\title{
Cascading Failure Phenomenon in the Multi-Stage Hydraulically Fractured Wells
}

\author{
Konstantin Sinkova ${ }^{\mathrm{a}}$, Dimitry Chuprakova ${ }^{\mathrm{a}}$, Maxim Chertov ${ }^{\mathrm{a}}$, Dean Willberga ${ }^{\mathrm{a}}$, Pavel Spesivtsev ${ }^{\mathrm{a}, *}$ \\ ${ }^{a}$ Schlumberger Moscow Research, 16A/3, Leningradskoe shosse, 125171 Moscow, Russia
}

\begin{abstract}
The phenomenon of cascading fracture failure during flowback and initial production from a horizontal multistage hydraulically fractured well is introduced, described, and investigated. First, a simplified analytical model of production from such well is built. This model allows evaluating a range of systems parameters through which the cascading failure evolves and performing a sensitivity study of this effect. Next, while keeping the physical model of the system relatively simple, the critical flow rates causing the motion of proppant pack in fractures are treated as random variables. This assumption brings the next level of sophistication to the model and allows demonstrating non-obvious effects. In particular, the well production rate losses due to cascading fracture failure are estimated. Finally, the proposed hypothesis is validated by conducting numerical simulations of flowback in the estimated conditions of cascading failure. As a practical outcome of this study, recommendations on the mitigation of the well productivity failure caused by cascading failure are formulated and discussed.
\end{abstract}

Keywords: multistage, hydraulic, fracturing, flowback, cascading, failure

\section{Introduction}

Over the last decades, horizontal well drilling and multistage hydraulic fracturing have become increasingly popular in the oilfield industry. Typically, the combination of 5 these technologies is required to produce oil and gas from low-permeability reservoirs at economic rates $[1,2,3,4]$. Created fractures hydraulically connect the well to a reservoir and substantially increase well productivity roughly proportional to the total number of created fractures. In

10 multistage fractured (MSF) wells, hydraulic fractures produce simultaneously from a reservoir to the same well and interact with each other via the and bottom-hole pressure in the lateral. Such an interactive fracture behavior may lead to interesting and, at a first glance, unexpected re15 sults, such as emergent behavior and cascading fracture failure. Cascading failure usually refers to the process in network system where failure of some parts causes the redistribution of load on other parts and triggers their failure due to increased load. Similarly, we define this phenomena as follows. A cascading failure is a process in a system of ${ }_{45}$ hydraulic fractures connected to the same well in which the failure of one or few fractures (for example, because of proppant flowback associated with excessive production rate) triggers the failure of other fractures and so on.

Cascading processes are well-known in many branches ${ }_{50}$ of applied mathematics. For example, cascading outage

\footnotetext{
* Corresponding author

Email addresses: ksinkov@slb.com (Konstantin Sinkov), dchuprakov@slb.com (Dimitry Chuprakov), mchertov@slb.com (Maxim Chertov), willberg2@slb.com (Dean Willberg), pspesivtsev@slb.com (Pavel Spesivtsev)
}

processes in power systems $[5,6,7]$ and cascading failures in temporal networks of interrelated tasks associated with project development [8] have been reported and investigated. To the best of our knowledge, such phenomena in producing MSF wells have not been reported in open literature. The closest domain where such phenomenon has been studied is modeling of hydraulic water or gas distributing networks $[9,10,11]$. However, there are private observations and speculations among oil and gas field professionals about the possibility of cascading fracture failure in such wells. Therefore, the objective of this paper is to investigate the interactive fracture behavior in MSF wells and mechanisms leading to their possible cascading failure.

\section{Key Questions}

As mentioned above, the effect of cascading fracture failure lacks description in the open oilfield industry literature. Hence, throughout this work we are aiming at addressing the following four fundamental questions:

1. What is cascading fracture failure?

2. Why does it evolve in horizontal multistage fractured wells?

3. Where and under which conditions does it occur, and where does it not occur?

4. How can we avoid this negative event?

\section{Cascading Fracture Failure Process}

Cascading failure of fractures in an MSF well is the uncontrolled sequential productivity damage by flow of many fractures, such that failure of the first fracture causes sub${ }_{55}$ sequent failure of other fractures in the well. Suppose that 
all producing fractures are damageable. We assume that these fractures might fail if one of the parameters (e.g., pressure or rate) exceeds certain value. In this paper, we $e_{115}$ refer to this parameter as critical (critical pressure or crit-

60 ical rate). For example, the fractures might have different critical flow rate, below which they can produce without fracture damage. After the flow rate exceeds this value, the fracture fails and stops producing fluid to the well. Such ${ }_{120}$ situation may occur, for example, as a result of destabi65 lization and washing out of the proppant from the nearwellbore region of this fracture. Under the high confining rock stress, the near-wellbore region unsupported by proppant will completely close and reduce fracture productivity ${ }_{125}$ to zero [12].

Consider a completed horizontal well equipped with a choke at surface and having multiple productive fractures created downhole. The mechanics of the cascading fracture failure process can be illustrated using the scenario presented in Figure 1. The wellhead choke allows

75 controlling the total production rate from the well. The ${ }_{130}$ fractures are different, so the critical parameter of failure is described by some distribution so that there is a varied failure resistance across the created fractures. The flow rate of fractures is induced by the pressure drop in the well

${ }_{80}$ with respect to the reservoir fluid pressure, also referred ${ }_{135}$ to as drawdown. Let us assume that initially (Figure 1,a) the surface choke is set in such a way that the well produces at a relatively small production rate $Q_{1}$. This total production rate $Q_{1}$ is the sum of contributions from $N$ hy85 draulic fractures, which produce with the same flow rate $Q_{1} / N$. At this rate, all fractures produce without any damage. Later, the choke is opened more, and the production rate is increased to $Q_{2}$ (Figure 1,b). After this change, the weakest fracture in the well is damaged and

90 stops producing (Figure 1,c). Due to the presence of a wellhead choke, the total flow rate decrease is translated into the decrease of the bottomhole pressure and increase of the drawdown. This mechanism increases the flow rate bringing it to the value $Q_{3}$ that gets close to $Q_{2}$. Due to

${ }_{95}$ the smaller number of producing fractures, it increases the ${ }^{140}$ producing rate at each remaining fracture, which becomes $Q_{3} /(N-1)>Q_{2} / N$ (Figure $\left.1, c\right)$. As a result of the production rate per active fracture ramping up, the critical flow rate is reached for two other fractures in the well (red arrows in Figure 1,c). As the choke opening increases, the ${ }^{145}$ well production rate continues increase leading in turn to increased drawdown for these fractures (Figure 1, $d$ ). Following this fracture, other fractures continue to fail one by one (Figure 1,e,f). This process of cascading failure may 105 impact either some of the fractures or all of them, so that in extreme cases the entire well productivity could be lost (Figure 1,f).

In this work, we investigate mechanics of interactive fracture behavior in a horizontal well during flowback and ${ }_{110}$ initial production. We evaluate conditions for triggering cascading fracture failure. The content of this paper is organized as follows. First, we explain the essence of the 150 cascading fracture failure phenomenon in MSF horizontal wells using simple illustrations. Next, we build a physically justified analytical model of well production enabled by a large set of productive but damageable fractures. This deterministic model allows predicting onset of cascading fracture failure for the given well, wellhead, fracture, and reservoir parameters. Next, we introduce probabilistic description of the MSF well. Using this description, we perform comprehensive study of the full system behavior and discuss implications of cascading failure events for the potential total loss of production. After this, we verify our analytical models by running accurate numerical simulations of flowback with consequent cascading failure using a rigorous numerical solver.

\section{Basic Physical Model}

Consider an L-shaped well with $N$ hydraulic fractures connected to its horizontal section and a choke at the wellhead (Figure 1). Assume that hydraulic fractures produce incompressible single-phase fluid at certain rates, depending on pressure drawdown. Because during initial production primarily water-like fluid is flowing back, the singlephase flow assumption can be justified. Individual fractures inflows $q^{k}$ are commingled into the total production rate at the wellhead and the choke:

$$
Q=\sum_{k=1}^{N} q^{k} .
$$

Under such assumptions, the relation between the total mass flow rate $Q$ and the pressure drop across choke $\Delta p_{c h}=p_{w h}-p_{w h d c}$ is given by (see, for example, [13])

$$
\begin{gathered}
Q=K C^{-1 / 2} \sqrt{\Delta p_{c h}} \\
K=\sqrt{2 C_{d}^{2} \rho A^{2}}, \quad C=\left(\left(\frac{d}{d_{c h}}\right)^{4}-1\right) .
\end{gathered}
$$

Here $p_{w h}$ is the wellhead pressure, $p_{w h d c}$ is the wellhead downstream choke pressure, $d_{c h}$ is the choke opening, $d$ is the pipe diameter, $A=\pi d^{2} / 4$ is the pipe cross-section area, and $\rho$ is the liquid density. Discharge coefficient $C_{d}$ describing irreversible friction losses is introduced into Equation (2) empirically. Factor $C \in[0, \infty]$ represents operational conditions at the choke defined by the chosen management strategy; $C=0$ corresponds to the fully open choke and $C=\infty$ corresponds to the fully closed choke.

Pressure drop in the wellbore is assumed to be dominated by the hydrostatic term. Accordingly, the bottomhole pressure $p_{b h}$ is shared between all fractures and given by the sum of the wellhead pressure $p_{w h}$ and the pressure drop at the vertical section of the well:

$$
p_{b h}=p_{w h}+\rho g h .
$$

Here $g$ is the gravity acceleration and $h$ is the depth of the horizontal well section relative to the wellhead. 
a)

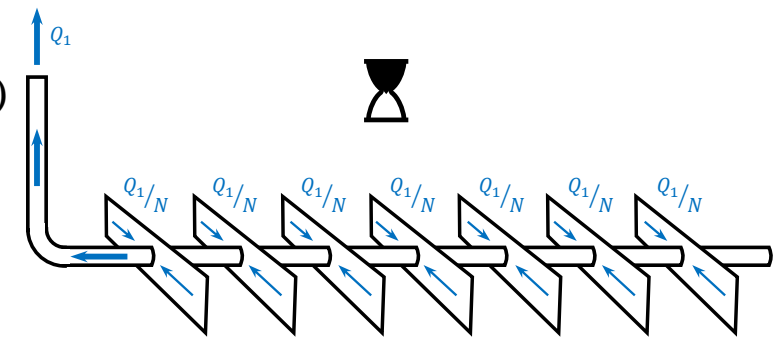

c)
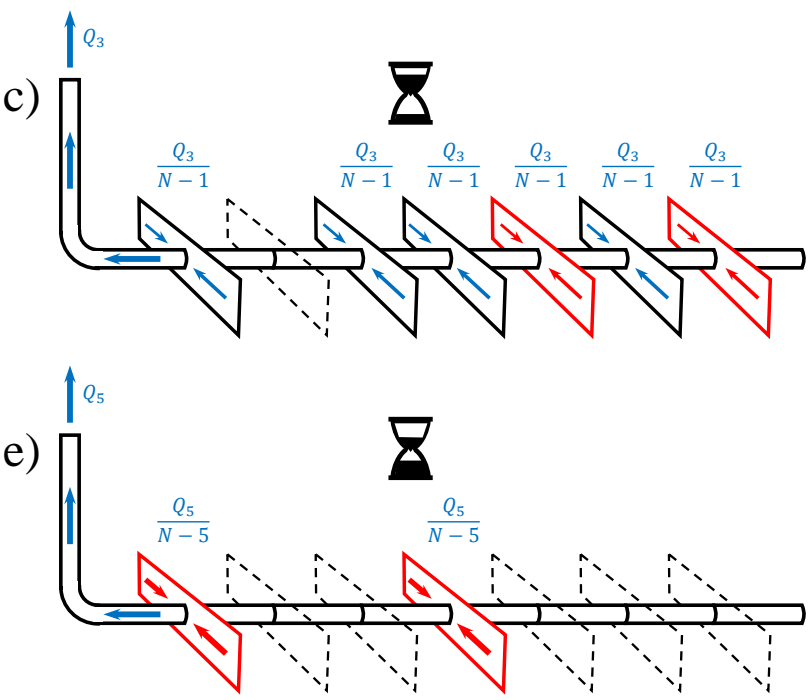
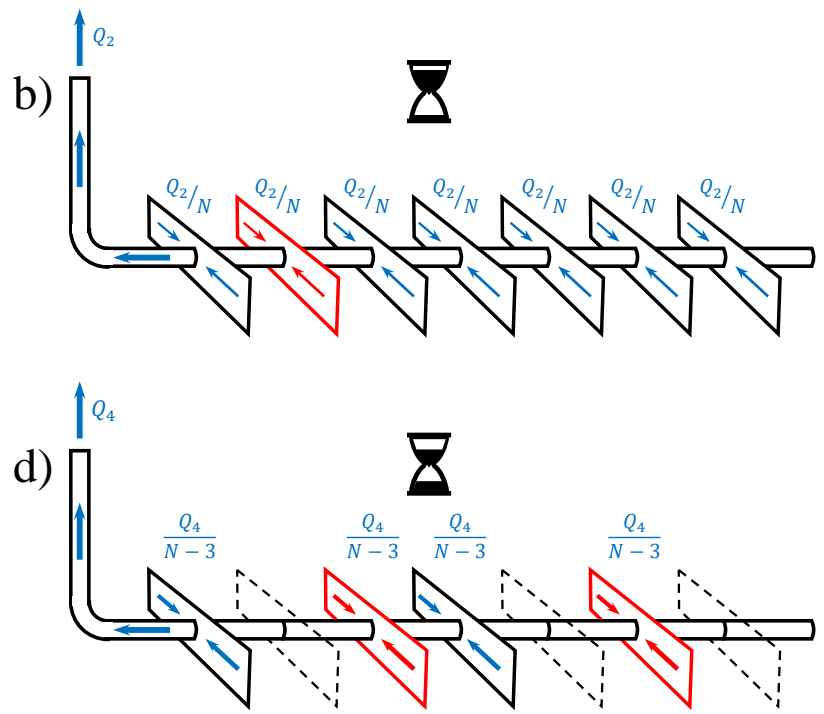

$Q_{6}=0$

f)

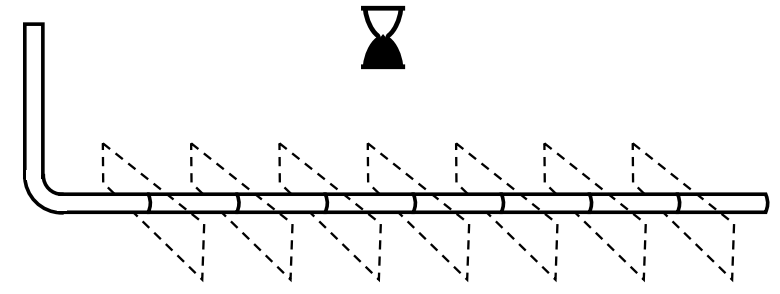

Figure 1: Illustration of cascading fracture failure process.

The inflow from the $k$-th fracture is

$$
q_{k}=J_{k}\left[p_{R}-p_{\mathrm{bh}}\right]
$$

where $J_{k}$ is the productivity index of the fracture, which can be estimated, for example, using the following Carter model [14]:

$$
J_{k}(t)=S_{k} \sqrt{\frac{k \phi c_{t}}{\pi \mu t}},
$$

where $S_{k}$ is the fracture surface, $k$ is the permeability of formation, $\phi$ is the porosity of formation, $c_{t}$ is the total compressibility of formation, $\mu$ is the producing fluid viscosity.

Special studies have been devoted to investigation of variations of rock permeability and pore pressure in rock formations [15, 16, 17]. In this model, we assume that matrix properties, such as permeability, porosity, in-situ stresses, and pore pressure, do not change significantly along the wellbore and fractures. Similarly, we assume similarity of geometrical and mechanical fracture characteristics within one multi-stage well. This assumption allows us to pay special attention to cascading effects in an interactive system of fractures rather than additional influence of rock heterogeneity, which may appear subsidiary. The effect of rock and fracture property variation can be considered in future studies.

An aggressive flowback strategy, when drawdown pressures and flow rates are high, can result in fracture damage. As mentioned above, the fracture conductivity can be damaged by different mechanisms that include, for example, washing of proppant out of the fracture, proppant crushing and embedment, fines migration, and tensile failure of rock and fracture faces. In this model, we assume that the $k$-th fracture maintains productivity as long as its rate is below some critical value, $q_{c}^{k}$ or, equivalently, as long as the bottomhole pressure is above some critical value, $p_{c}^{k}$ defined via

$$
p_{c}^{k}=p_{R}-q_{c}^{k} / J_{k} .
$$

Then, fractures can experience complete and irreversible loss of productivity if the failure criterion is met:

$$
q^{k}>q_{c}^{k}
$$

or, in terms of pressure

$$
p_{b h}<p_{c}^{k}
$$




\section{Probabilistic description}

The merit of probabilistic description is that it is not tures. A modern multistage hydraulically fractured well can be connected with over a hundred hydraulic fractures, and it can be impractical to characterize parameters of every fracture by either measurements or simulations or ead, it can be more convenient to specify probabilities of some parameters to stay within certain ranges established statistically. Such probabilistic description allows deriving response of the large system of hydraulically interacting fractures in terms of expected values and variances of parameters.

To formulate the probabilistic description, it is convenient to further simplify physical model described in section 2. Productivity indices of all fractures are assumed to be the same, $J_{k}=J_{0}, \forall k$. Probabilistic analysis is perduring relatively short time interval, such that productivity index $J_{0}$ is assumed to be constant despite the $\sqrt{t}$ dependency typically observed in the fracture productivity behavior [14]. We also suppose that during startup ${ }^{210}$ of the well the choke opening increases and the bottomhole pressure decreases immediately after the next choke opening and remains constant in between the choke size changes, which is typical to a hydraulically fractured well flowback scenario. Each fracture is considered to operate ${ }_{215}$ with the constant productivity index $J_{0}$ if its individual rate $q^{k}$ is less than some critical value $q_{c}^{k}$ and totally and irreversibly lose productivity if $q^{k}$ at some moment of time exceeds $q_{c}^{k}$.

Under these assumptions, the inflows are modeled by b2 $_{22}$ the following relation:

$$
q^{k}=J_{0} \theta\left(p_{b h}-p_{c}^{k}\right)\left(p_{R}-p_{b h}\right) .
$$

Here $\theta(\cdot)$ is the Heaviside step function and $p_{c}^{k}=p_{R}-$ $q_{c}^{k} / J_{0}$ is the critical bottomhole pressure for fracture fail- ${ }^{225}$ ure.

Using Equation (2) and (9), one can obtain the following system of algebraic equations describing steady states of the well:

$$
\begin{gathered}
Q=J_{0}\left(p_{R}-p_{b h}\right) \sum_{k=1}^{N} \theta\left(p_{b h}-p_{c}^{k}\right), \\
Q=K C^{-1 / 2} \sqrt{p_{b h}-p_{b h 0}} .
\end{gathered}
$$

It is important to note that during flowback the well is cer-235 tainly not in steady state regime so that different transient effects (e.g. pressure and rate jumps) can take place as a result of changes in boundary conditions (e.g. changes of choke opening or pressure at the wellhead). These effects can also have impact on the evolution of cascading failure ${ }_{240}$ process and can be investigated in future research. We demonstrate that cascading failure can be described and investigated using this steady-state formulation.

Here $p_{b h 0}=p_{w h d c}+\rho g h$ is the bottomhole pressure for the fully opened choke and $Q=\sum_{k=1}^{N} q^{k}$. Sorting 245 the sequence $p_{c}^{k}$ in increasing order, one can obtain new sequence $p_{c}^{(k)}$. Then, for each interval of the bottomhole pressure $\left(p_{c}^{(k)}, p_{c}^{(k+1)}\right), k=\overline{1, N}, p^{(N+1)}=p_{R}$ system $(10)$ is reduced to a quadratic equation with respect to $p_{b h}$. One of the roots of this equation always exceeds reservoir pressure and thus corresponds to negative total flow rate. Equations (10) describe production flows and allow positive flow rates only. Accordingly the discussed root has no physical meaning in our problem. Another root in the dimensionless form is given by

$$
p_{b h}^{k}=1+\frac{1}{2 C \Pi k^{2}}-\sqrt{\frac{1}{4\left(C \Pi k^{2}\right)^{2}}+\frac{1}{C \Pi k^{2}}\left(1-p_{b h 0}\right)} .
$$

Here $k=\overline{1, N}$, reservoir pressure $p_{R}$ and rate of $N$ working fractures at minimum possible bottomhole pressure $N J_{0}\left(p_{R}-p_{b h 0}\right)$ are chosen as pressure and flow rate scales and

$$
\Pi=\frac{J_{0}^{2} p_{R}}{2 C_{d}^{2} \rho A^{2}} .
$$

The candidate root $p_{b h}^{k}$ must lie in the corresponding interval $\left(p_{c}^{(k)}, p_{c}^{(k+1)}\right)$ to describe actual steady state of the well. Accordingly, we will say that for the given choke opening, the well has steady state if there exists at least one $k^{*}=\overline{1, N}$ such that $p_{b h}^{k^{*}} \in\left(p_{c}^{\left(k^{*}\right)}, p_{c}^{\left(k^{*}+1\right)}\right)$. Otherwise, it is said that the well has no steady states.

As we suppose that during startup of the well the choke opening increases and the bottomhole pressure decreases, the system reaches the steady state with the maximum value of pressure first and stays there until further adjustment of choke opening. Consequently, we study only the steady state with the maximum value of pressure and correspondingly the maximum number of producing fractures $k^{*}$.

Figure 2 graphically shows the example solutions of the system of equations (10). At the left plot the critical pressures are such that only one candidate root $p_{b h}^{5}$ belongs to the corresponding interval $\left(p_{c}^{(5)}, p_{c}^{(6)}\right)=1$ and, therefore the well has a steady state with the maximum number of fractures $k^{*}=5$ producing. At the center plot, for different choice of critical pressures there are three suitable candidate roots, all with number of producing fractures less than the total number and the well has a steady state with $k^{*}=4$. At the right plot, for slightly different choice of critical pressures there are no candidate roots within corresponding intervals and the well has no steady states. Depending on the distribution of critical pressures within the same interval, we can observe substantially different situations for the constant choke size from maximum number of fractures producing at the left plot to entire well collapse at the right plot. Moreover, the steady states are extremely sensitive to the choice of critical pressures. It is difficult to estimate particular values of critical pressures prior to the well start up. Therefore, instead of studying particular configurations of critical rates and pressures, we suggest treating them as random variables. 

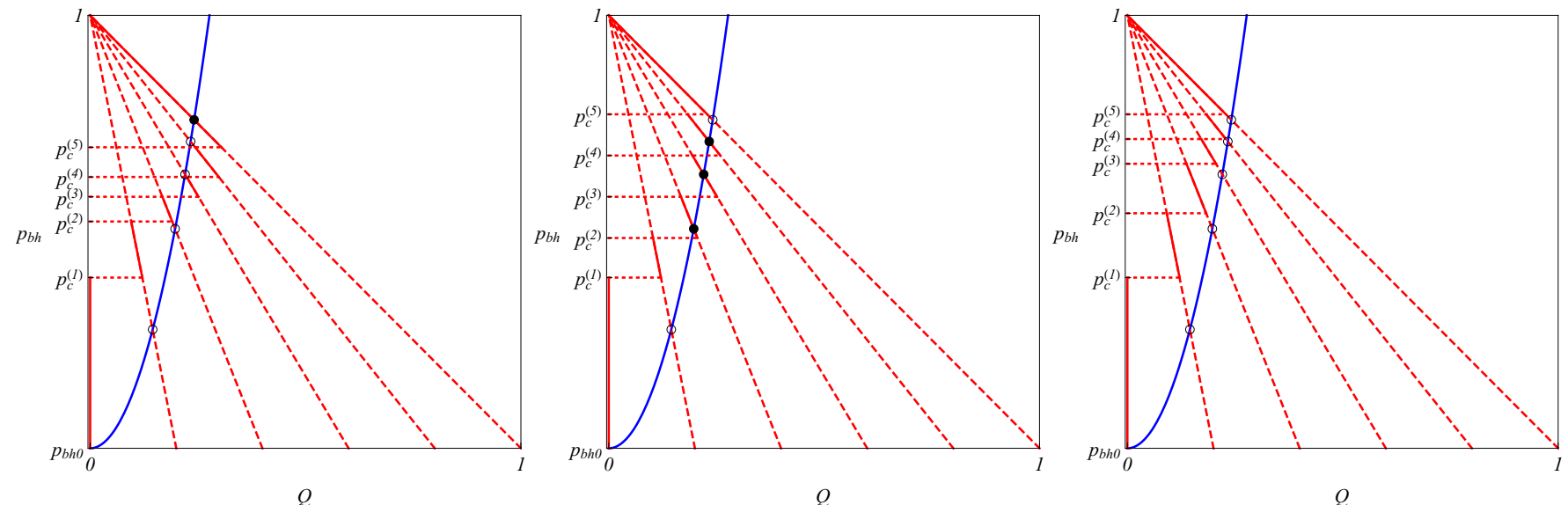

Q

$Q$

Figure 2: Graphical representations of the system (10) for $N=5$ and three different sets of $p_{c}^{(k)}$ on the $Q-p_{b h}$ plane. Choke size is the same for all plots. Lines: the dependency of rate on the bottomhole pressure representing wellbore and choke (solid blue), linear inflow performance relations, corresponding to $1,2, \ldots, 5$ fractures producing (dashed red) and the resulting dependency of the total flow rate from fractures on the bottomhole pressure (solid red). Points: candidate solutions with $p_{b h}^{k}$ outside (circles, crossing of solid blue and dashed red lines) and inside (disks, crossing of solid lines) corresponding intervals $\left(p_{c}^{(k)}, p_{c}^{(k+1)}\right)$. The actual steady-state solution is the disk with the highest pressure.

Table 1: Physical parameters.

\begin{tabular}{|c|c|c|c|c|c|c|}
\hline Parameter & Symbol & Unit & Case 1 & Case 2 & Case 3 & Case 4 \\
\hline Reservoir pressure & $p_{R}$ & psi & \multicolumn{3}{|c|}{7000} & 7107 \\
\hline Fluid density & $\rho$ & $\mathrm{kg} / \mathrm{m}^{3}$ & \multicolumn{3}{|c|}{1000} & 1000 \\
\hline Wellbore diameter & $d$ & inch & \multicolumn{3}{|c|}{4.0} & 4.7 \\
\hline Discharge coefficient & $C_{d}$ & - & \multicolumn{3}{|c|}{0.85} & 0.85 \\
\hline True vertical depth & $h$ & $\mathrm{ft}$ & \multicolumn{3}{|c|}{9843} & 9843 \\
\hline Wellhead downstream choke pressure & $p_{w h d c}$ & psi & \multicolumn{3}{|c|}{435} & 1450 \\
\hline Average fracture productivity index & $J_{0}$ & $\mathrm{bbl} /($ day $\cdot \mathrm{psi})$ & \multicolumn{3}{|c|}{$2.5 \cdot 10^{-2}$} & $6.7 \cdot 10^{-2}$ \\
\hline Minimum critical volumetric flow rate & $\hat{q}_{c}^{\text {min }}$ & $\mathrm{bbl} /$ day & 20.0 & 10.0 & 10.0 & 3.3 \\
\hline Maximum critical volumetric flow rate & $\hat{q}_{c}^{\max }$ & $\mathrm{bbl} /$ day & 30.0 & 30.0 & 20.0 & 87.0 \\
\hline Number of fractures & $N$ & - & \multicolumn{3}{|c|}{100} & 100 \\
\hline
\end{tabular}

Table 2: Dimensionless parameters.

\begin{tabular}{|c|c|c|c|c|c|}
\hline Parameter & Symbol & Case 1 & Case 2 & Case 3 & Case 4 \\
\hline Minimum bottomhole pressure & $p_{b h 0}$ & \multicolumn{3}{|c|}{0.68} & 0.80 \\
\hline Conductivity number & $\Pi$ & \multicolumn{3}{|c|}{$2.26 \cdot 10^{-8}$} & $8.77 \cdot 10^{-8}$ \\
\hline Minimum critical pressure & $p_{-}$ & 0.83 & 0.83 & 0.89 & 0.82 \\
\hline Maximum critical pressure & $p_{+}$ & 0.89 & 0.94 & 0.94 & 0.99 \\
\hline
\end{tabular}

Now consider the critical pressures $p_{c}^{k}$ to be identical random variables with the probability density function $f$ and the cumulative distribution function $F$. The sequence of re-ordered critical pressures $p_{c}^{(k)}$ becomes the sequence of order statistics of the statistical sample $p_{c}^{k}$. Realization of $k$-th steady state with $k$ fractures working is now a random event. Below we will calculate the probability $P_{k}$ associated with it.

The joint probability distribution function of order statis- $\operatorname{tics} p_{c}^{(k)}, \ldots, p_{c}^{(N)}$ is calculated as follows [18]

$$
\begin{aligned}
f_{k}\left(p_{c}^{(k)}, \ldots, p_{c}^{(N)}\right)= & \frac{N !}{(k-1) !} F\left(p_{c}^{(k)}\right)^{k-1} \times \\
& \quad \times \prod_{i=k}^{N} f\left(p_{c}^{(i)}\right) \theta\left(p_{c}^{(i+1)}-p_{c}^{(i)}\right) .
\end{aligned}
$$

Let $\mathcal{A}(k, C)$ be the event describing that $k$ fractures are producing at choke opening $C$. The probability of $\mathcal{A}(k, C)$ 
(i.e., the $k$-th steady state is reached) is given by

$$
\begin{aligned}
& P_{k}=P(\mathcal{A}(k, C))= \\
& =\underbrace{\int \cdots \int} \prod_{i=k}^{N} d p_{c}^{(i)} \times f_{k}\left(p_{c}^{(k)}, \ldots, p_{c}^{(N)}\right) \times \\
& \quad \times \theta\left(p_{b h}^{k}-p_{c}^{(k)}\right) \theta\left(p_{c}^{(k+1)}-p_{b h}^{k}\right) \times \\
& \times \prod_{i=k+1}^{N}\left[\theta\left(p_{c}^{(i)}-p_{b h}^{i}\right)+\theta\left(p_{b h}^{i}-p_{c}^{(i+1)}\right)\right] .
\end{aligned}
$$

Here the integration is carried out over any suitable interval containing the support of $f$. The product of the first two theta-functions shows that the $k$-th candidate pressure (11) is between the $k$-th and $k+1$-th critical pressures. The rest factors show that all candidate pressures larger than $k$-th lie outside of corresponding intervals. Calculation of $P_{k}$ is outlined in Appendix A.

Note that the integral in Equation (13) depends only on the values of candidate pressures $p_{b h}^{k}$. This means that the probability calculations and physical model are well separated. Therefore, it is possible to use a more sophisticated description of wellbore flow as compared to Equation (3) and/or inflow performance relationship different from Equation (9) to produce the sequence of $p_{b h}^{k}$.

The bottomhole pressure, total flow rate, and number of surviving fractures are now random variables with some probability distributions. In the next section, we will qualitatively analyze these distributions. For illustration purposes, we consider only the case of the uniform distribution $f$. The probability density function $f(x)$ and cumulative distribution $F(x)$ in this case are given by

$$
\begin{gathered}
f(x)=\frac{1}{p_{+}-p_{-}} \theta\left(x-p_{-}\right) \theta\left(p_{+}-x\right), \\
F(x)=\frac{x-p_{-}}{p_{+}-p_{-}} \theta\left(x-p_{-}\right) \theta\left(p_{+}-x\right)+\theta\left(x-p_{+}\right) .
\end{gathered}
$$

However, the procedure presented in Appendix A is valid for an arbitrary probability distribution.

\section{Qualitative analysis}

We expect the following schematic picture of different probability distributions under varying choke size. If the maximum candidate pressure $p_{b h}^{N}$ is above the value $p_{+}$the well is operated under safe conditions and all $N$ fractures are producing, $P_{N}=1$. These conditions are met for sufficiently small choke openings. After choke size exceeds certain value corresponding to the condition $p_{b h}^{N}=p_{+}$all285 steady states with $k=\overline{0, N}$ fractures producing are possible. Probability of the entire system collapse $P_{0}$ grows as the choke size increases because the bottomhole pressure decreases and becomes more probable to be below critical pressure for some fraction of fractures. Finally, $P_{0}$ becomes ${ }^{290}$ equal to unity at the opening defined by $p_{b h}^{N}=p_{-}$then the
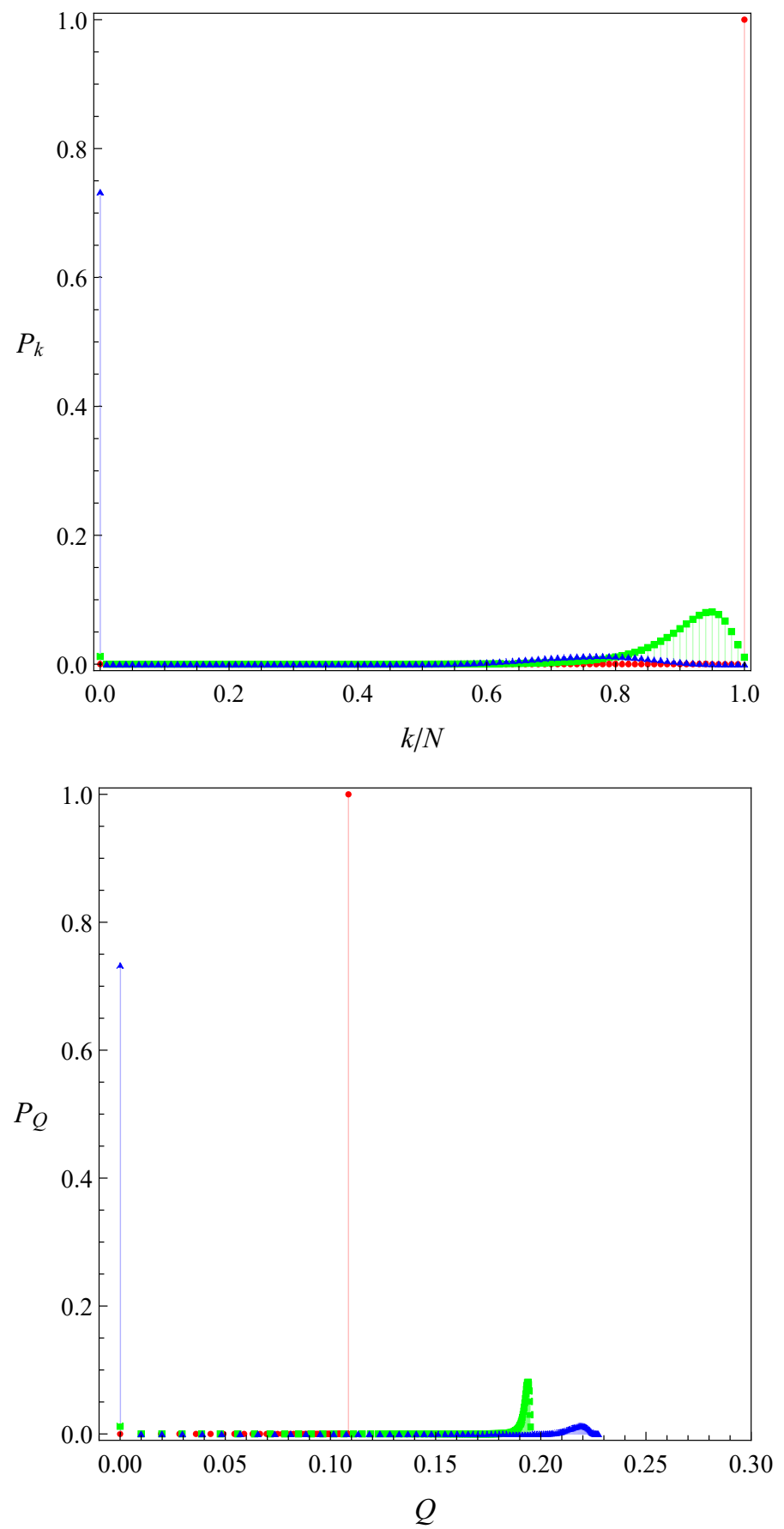

Figure 3: Probability distributions for the number of producing fractures (top) and the total rate (bottom). The parameters used are summarized in Table 1 , case 1 . The choke sizes $8 / 64^{\prime \prime}$ (red disks), $11 / 64^{\prime \prime}$ (green squares), 12/64" (blue triangles).

bottomhole pressure is certainly below critical pressure for all fractures.

Figure 3 illustrates the evolution of probability distributions of the number of producing fractures and the total flow rate. For the smallest choke size, the well is in the safe zone (all fractures produce) but the drawdown and the rate are low. For the intermediate choke opening, the drawdown is higher but the probability of failure of 
several fractures is non-zero. For the largest choke size, all fractures lose conductivity with very high probability, however, if some of them are not damaged, the well total ${ }_{330}$ production is relatively high.

Another way to analyze the behavior of the well is the analysis of the moments of the number of producing fractures $k$ and the total flow rate $Q$. For such analysis, introduce the expected values and variances

$$
\begin{gathered}
\mu_{k}=\sum_{k=1}^{N} P_{k} k, \quad \sigma_{k}^{2}=\sum_{k=1}^{N} P_{k} k^{2}-\mu_{k}^{2}, \\
\mu_{Q}=\sum_{k=1}^{N} P_{k} Q_{k}, \quad \sigma_{Q}^{2}=\sum_{k=1}^{N} P_{k} Q_{k}^{2}-\mu_{Q}^{2} .
\end{gathered}
$$

Here the rate $Q_{k}$ corresponds to $k$ producing fractures. Using the same characteristic scales as in derivation of Equation (11) ( $p_{R}$ for pressure and $N J_{0}\left(p_{R}-p_{b h 0}\right)$ for rate) one can obtain the following equation for dimensionless rates $Q_{k}$ :

$$
Q_{k}=\frac{1-p_{b h}^{k}}{1-p_{b h 0}} \frac{k}{N}
$$

Figure 4 shows plots of the parametric curves $\left(\sigma_{k}\left(d_{c h}\right)\right.$, $\left.\mu_{k}\left(d_{c h}\right)\right),\left(\sigma_{Q}\left(d_{c h}\right), \mu_{Q}\left(d_{c h}\right)\right)$ and $\mu_{k}\left(d_{c h}\right), \mu_{Q}\left(d_{c h}\right)$. Expectation of the number of producing fractures monotonically decreases with increase of the choke size. If our goal is to protect the maximum number of fractures, the only strategy is to keep the bottomhole pressure far from the dangerous range. If our goal is the high flow rate or both high rate and number of producing fractures, the right strategy of managing the choke opening is not so obvious. f we are concerned with the rate only, the natural desire is to get the maximum rate (characterized by the expected value $\mu_{Q}$ ) at the minimum uncertainty (characterized by the variance $\sigma_{Q}$ ). As one can see from Figure 4 , center it is not possible to reach both extrema simultaneously (case 2). A possible solution may be to specify some acceptable level of absolute or normalized variance that we could tolerate and optimize the $\mu_{Q}$ under constrain of $\sigma_{Q}$ bounded by this tolerance.

Another observation can be made using Figure 4, right. rate might correspond to the number of producing fractures notably lower than the total number of fractures $N$. One can interpret this fact in the following way. It is possible to choose aggressive choke management strategy systematically and damage recently created fractures without obvious manifestation of this damage in the surface flow rate.

\section{Correction of expectations based on observa- tions \& choke management policy}

In section 3 we formulated some expectations about the $\mathrm{s}_{350}$ state of the well for a given choke size in terms of associated probabilities. These expectations do not take into account information that may be acquired during the actual process of well startup. Consider the well that already passed through the set of $M$ states characterized by the value of choke size $d_{c h}^{m}$ or the values of $C_{m}, m=\overline{1, M}$. Using the recorded values of the total flow rate at the previous choke sizes and the value of an individual fracture conductivity $J_{0}$ (supposed to be known), one can obtain the numbers $k_{m}$ of surviving fractures corresponding to the $C_{m}$. Below we will try to use this information about the past performance of the well to correct our expectations about its future behavior.

The probability of the event that $k=k_{0}$ fractures will produce at choke opening $C=C_{0}$ given that $k_{1}$ fractures produced at choke opening $C_{1}, k_{2}$ at $C_{2}, \ldots, k_{M}$ at $C_{M}$ is the conditional probability given by

$$
\begin{aligned}
P_{\left(k_{0}, C_{0}\right) \mid\left(k_{1}, C_{1}\right), \ldots,\left(k_{M}, C_{M}\right)}= & \\
= & P\left(\mathcal{A}\left(k_{0}, C_{0}\right) \mid \bigcap_{m=1}^{M} \mathcal{A}\left(k_{m}, C_{m}\right)\right)= \\
= & \frac{P\left(\bigcap_{m=0}^{M} \mathcal{A}\left(k_{m}, C_{m}\right)\right)}{P\left(\bigcap_{m=1}^{M} \mathcal{A}\left(k_{m}, C_{m}\right)\right)} .
\end{aligned}
$$

Both numerator and denominator of the fraction in Equation (15) are defined similarly as in Equation (13):

$$
\begin{aligned}
& P\left(\bigcap_{m=m_{0}}^{M} \mathcal{A}\left(k_{m}, C_{m}\right)\right)= \\
& =\underbrace{\int \cdots \int}_{N-k_{m_{0}}+1} \prod_{i=k_{m_{0}}}^{N} d p_{c}^{(i)} \times f_{k_{m_{0}}}\left(p_{c}^{\left(k_{m_{0}}\right)}, \ldots, p_{c}^{(N)}\right) \times \\
& \quad \times \prod_{m=m_{0}}^{M}\left[\theta\left(p_{b h}^{k_{m}}\left(C_{m}\right)-p_{c}^{\left(k_{m}\right)}\right) \theta\left(p_{c}^{\left(k_{m}+1\right)}-p_{b h}^{k_{m}}\left(C_{m}\right)\right) \times\right. \\
& \left.\quad \times \prod_{i=k_{m}+1}^{N}\left[\theta\left(p_{c}^{(i)}-p_{b h}^{i}\left(C_{m}\right)\right)+\theta\left(p_{b h}^{i}\left(C_{m}\right)-p_{c}^{(i+1)}\right)\right]\right] .
\end{aligned}
$$

Here $m_{0}=0$ for the numerator and $m_{0}=1$ for the denominator in Equation (15). Each factor in the outer product is similar to the product of theta-functions in Equation (13). Note that here we should distinguish the candidate bottomhole pressure corresponding to the different choke sizes. From the assumption that the choke size increases in time, it follows that $C_{m+1}<C_{m}$ and $p_{b h}^{i}\left(C_{m+1}\right)<p_{b h}^{i}\left(C_{m}\right), \forall i, m$. Because the fractures lose productivity irreversibly, the number of surviving fractures in future is always smaller or equal to the number of fractures surviving in the past so that $k_{m} \leq k_{m+1}, \forall m$.

Calculation of $P_{\left(k_{0}, C_{0}\right) \mid\left(k_{1}, C_{1}\right), \ldots,\left(k_{M}, C_{M}\right)}$ is outlined in Appendix B. A noteworthy result of this calculation is that under assumptions given above, $P_{\left(k_{0}, C_{0}\right) \mid\left(k_{1}, C_{1}\right), \ldots,\left(k_{M}, C_{M}\right)}=$ 

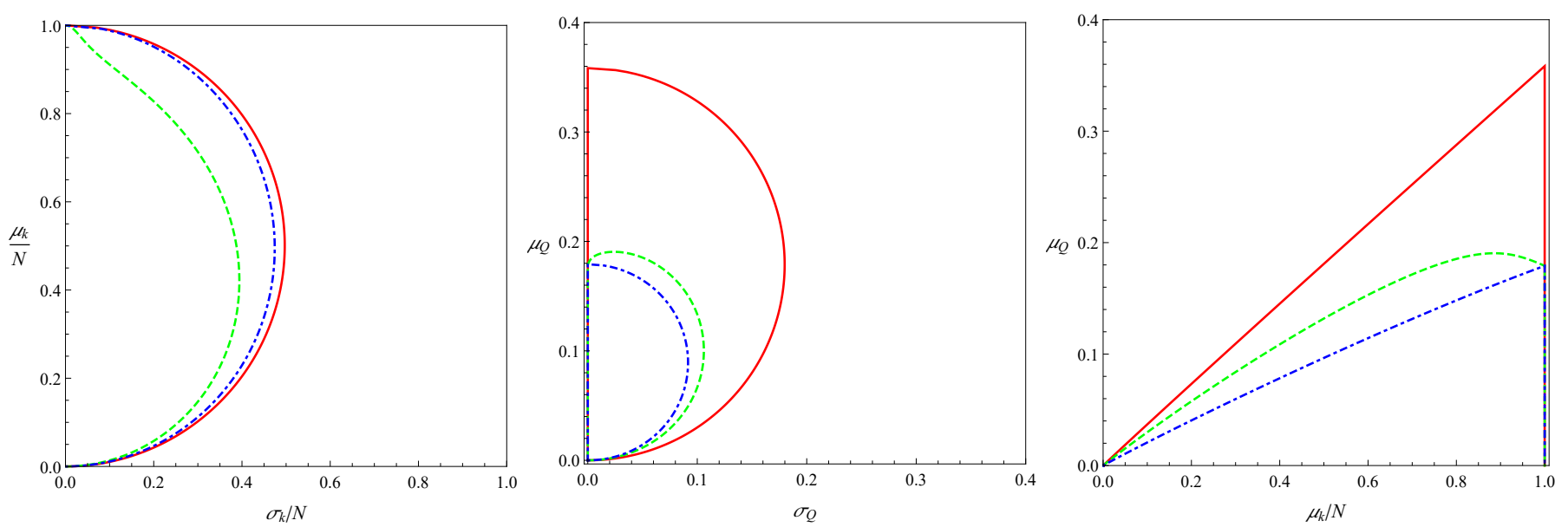

Figure 4: Parametric plots mean value vs. dispersion for the number of producing fractures (left) and the total production rate (center), the mean rate vs. mean number of producing fractures (right). The parameters used are given in Table 1 , cases 1 (solid red), 2 (dashed green), and 3 (dash-dotted blue). The maximum flow rate for the case 2 (dashed green) corresponds to the number of producing fractures significantly lower than the total number of fractures.

$P_{\left(k_{0}, C_{0}\right) \mid\left(k_{1}, C_{1}\right)}$. This means that all probability distributions are affected only by the last observed state of the ell. In other words, it does not matter how exactly (in what particular order and by what sequence of choke sizes) $)_{370}$ fractures have been damaged. Only the most recent observation helps to correct our future expectations about the well behavior.

The independence of the conditional probability on the previous states, except the last one, means that the process375 of well evolution is Markovian. This allows us to apply to the problem of proper choke management strategy a formalism of Markov decision processes (MDP) [19, 20].

We will refer to the pair (number of intact fractures, current choke parameter) as the state of well $s=(k, C)$. $\operatorname{In}_{380}$ the subsequent derivations, we consider the choke parameter $C$ to be from the finite set $\mathbb{C}_{L}=\left\{C_{l}\right\}_{l=1}^{L}$, for example induced by uniform step of choke size adjustment. This assumptions significantly simplifies problem statement. Let us denote the set of all possible states $\mathbb{S}=\mathbb{Z}_{N+1} \times \mathbb{C}_{L^{385}}$ and the set of available at the state $s$ actions $a$ (choke parameters adjustments) $\mathbb{A}_{s}=\{a \in \mathbb{C}: a \leq C\}$. Probabilities $P_{s s^{\prime}}^{a}$ of transition from state $s=(k, C)$ to state $s^{\prime}=\left(k^{\prime}, C^{\prime}\right)$ under action $a$ have been calculated in the previous section $P_{s s^{\prime}}^{a}=P_{\left(k^{\prime}, C^{\prime}\right) \mid(k, C)}$. Note that $P_{s s^{\prime}}^{a}=390$ $0, \forall k^{\prime}>k, \forall C^{\prime} \neq a, \forall a>C$. Finally, let us define an immediate reward function of the transition $s \rightarrow s^{\prime}$ under action $a$ as a difference between total flow rate at the states $s^{\prime}$ and $s, r_{s s^{\prime}}^{a}=Q_{k^{\prime}}\left(a=C^{\prime}\right)-Q_{k}(C)$. The problem of MDP is to find a policy $\Pi: \mathbb{S} \rightarrow \mathbb{A}_{s}$ maximizing $_{395}$ expected value of sum of the random immediate rewards

$$
V=\sum_{t=1}^{L} r_{s_{t} s_{t+1}}^{a_{t}}
$$

Here actions are chosen according to policy, e.g., $a_{t}=$ $\Pi\left(s_{t}\right)$. The horizon of planning is finite and equal to $L$ because we assume that the set of possible choke sizes is finite and choke opening can only increase in time. The problem considered is equivalent to maximization of the final total flow rate, one of the possible goals discussed in the section 4.

We apply the well known value iteration algorithm [21] to the MDP problem described above. After the policy is found, evaluation of the number of producing fractures is required to determine the current state $s_{t}$ and define the next action $a_{t}$. Throughout the paper, we use the assumption that the productivity index of the individual fractures are equal. Accordingly, the number of producing fractures may be calculated using the measured flow rate. This assumption is essential for application of MDP to our problem because it reflects fundamental assumption of MDP formalism that the environment is fully observable and the current state completely characterizes the process.

Figure 5 graphically illustrates the example of optimal policy. As one can note, for the majority of states, the optimal policy recommends keeping choke opening constant. In particular, for the choke sizes larger than $20 / 64^{\prime \prime}$, there are no observed number of producing fractures such that any action will increase the expected value of the final flow rate. However, for the lower openings and relatively high number of surviving fractures, it is reasonable to increase the choke further. Note that the increase of the choke by more than one step at once is never optimal. This conclusion seems to be natural and may be interpreted as follows. Choke opening increasing not only causes growth of flow rate but also allows gaining information about statistics of critical rates. Cautious choke opening in small steps helps collect the information gradually without additional risk of damaging fractures and utilize it at the next steps. 


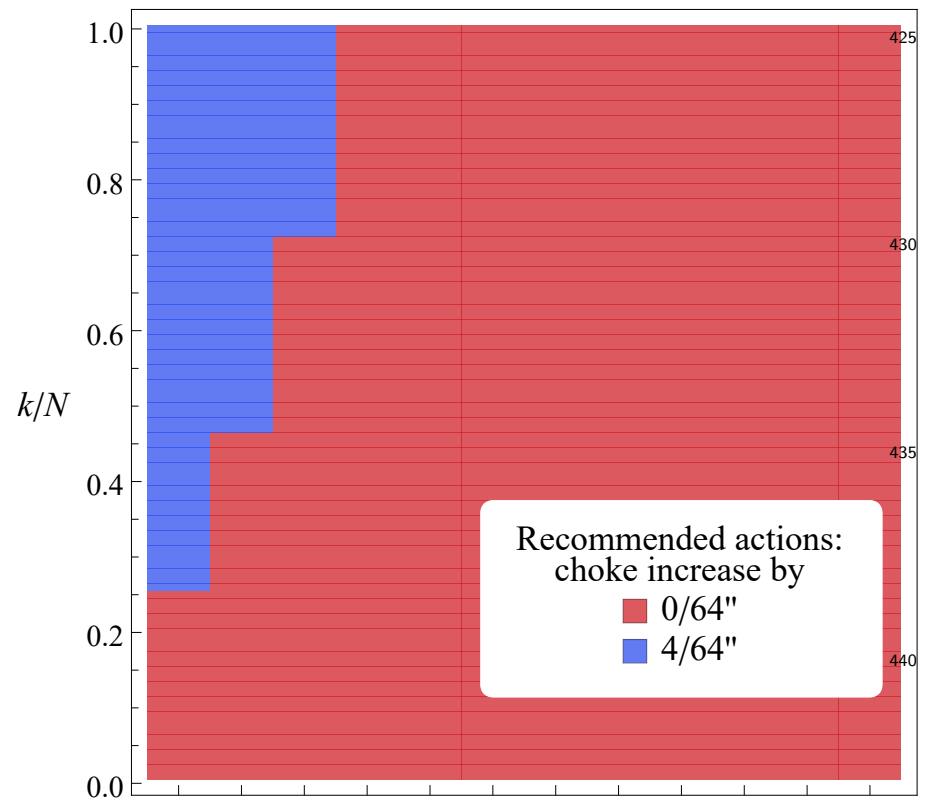

8. 12. 16. 20. 24. 28. 32. 36. 40. 44. 48.52.

$d_{\mathrm{ch}}[1 / 64 "]$

Figure 5: Choke management policy for the parameters listed in Table 1 , case 4 , and the range of choke parameters $\mathbb{C}_{L}$ corresponding to openings $8 / 64^{\prime \prime}, 12 / 64^{\prime \prime}, \ldots, 52 / 64^{\prime \prime}$. Each point of the diagram corresponds to state $s$, current choke opening is the horizontal coordinate and observed number of producing fractures relative to total number of fractures is the vertical coordinate. Color shows the optimal action for the state, either increase choke opening for some value (blue) or keep it constant (red).

\section{Numerical and analytical simulations}

For additional illustration of cascading failure in the system of interacting hydraulic fractures and to compare predictions of statistical and deterministic approach, we performed numerical and analytical modeling of the fracture- ${ }^{460}$ ellbore system as shown in Figure 1. The models simulated single-phase incompressible fluid flow in a horizontal well connected with 100 hydraulic fractures with parameters similar to case 4 in Table 1 . The simulated well has a $9843 \mathrm{ft}$ long vertical section and $6562 \mathrm{ft}$ long horizon- ${ }^{465}$

410 tal section. The fractures are placed equidistantly along the horizontal section and have identical spatial dimensions (66 ft height, $328 \mathrm{ft}$ half-length). All fractures are assumed having the constant $5 \mathrm{~mm}$ width and uniformly propped with permeability of the proppant pack equal to ${ }^{470}$

4151440 darcy. Rates of inflows from the reservoir into each cell element of the fractures are proportional to the difference between the fluid pressure in the cell and far-field reservoir pressure. Selected fracture conductivity is high enough to neglect pressure variation along the fracture. ${ }^{475}$

${ }_{420}$ As a result, all fractures effectively have the same constant productivity index $J_{0}$. The initial reservoir pressure is 7107 psi. The wellhead pressure downstream choke (WHDCP) was fixed at 1450 psi. The pressure drop and associated fluid production are controlled by gradual open- ${ }^{480}$ ing of the wellhead choke from $3 / 64$ " to $28 / 64$ ". We were prescribing different critical velocity or, equivalently, different critical flow rate for each fracture in the range from $3.3 \mathrm{bbl} /$ day to $87.0 \mathrm{bbl} /$ day.

In the numerical model, we coupled the model of the transient flows in a well, described in [22], with the numerical models of individual fractures [23]. Each fracture was represented by a grid of conductive cells with fixed width simulating a porous medium. Single-phase incompressible flow in these porous cells was described by a standard Darcy's equation [24] with constant viscosity and permeability of the proppant pack. If the flow rate from the fracture exceeded the critical value, all cells connecting the fracture to the well were updated with zero conductivity. The well and fracture models were coupled using iterative algorithm based on Picard iterations (or fixed point iterations method) [25] that ensured balance of pressures and flow rates between them within certain tolerance. More detailed description of the numerical model and its application to simulation of the cascading failure problem can be found in $[23,26]$. The problem of pressure-rate coupling between well and fractures has been closely investigated in [27], including study of accuracy and influence of different modifications of algorithm on convergence of fixed point iterations.

In the analytical model, we performed fine timestepping such that there could be more time steps between choke changes than total number of fractures and all fractures could potentially lose their productivity in the cascading failure sequence after a single choke increase. At a given timestep, the model would either increase the choke if it is prescribed by the choke schedule or reduce the number of producing fractures based on the comparison of the current flow rate and critical ones. Every time step also updates flow rates and bottomhole pressure. This ensures that immediate increase of production caused by the choke increase is recorded before some of the fractures are failed and there is a non-zero response time of fracture conductivity to the choke change. The analytical model neglects viscous pressure losses along the well, which in case of incompressible fluid allows defining updates of flow rates and bottomhole pressure as explicit functions of choke size and number of fractures.

Figure 6 represents the comparison of the numerical and analytical models for the specific choice of critical fracture flow rates. In Figure 6 the critical flow rates monotonically and uniformly increase from the lowest (3.3 bbl/day) to the highest $(87.0 \mathrm{bbl} /$ day $)$. The wellhead choke is gradually opened from $3 / 64$ " to $28 / 64$ " during the first 25 hours, making a step-wise increase of $1 / 64$ " every hour. Note that the choice of the uniform distribution of critical velocities in deterministic approach is made because a) it has the same mean as uniform random distribution and b) it resembles the uniform probability density function used in the statistical description above. However, it is not guaranteed that evolution with time or with choke size of this specific realization of the ensemble of critical frac- 

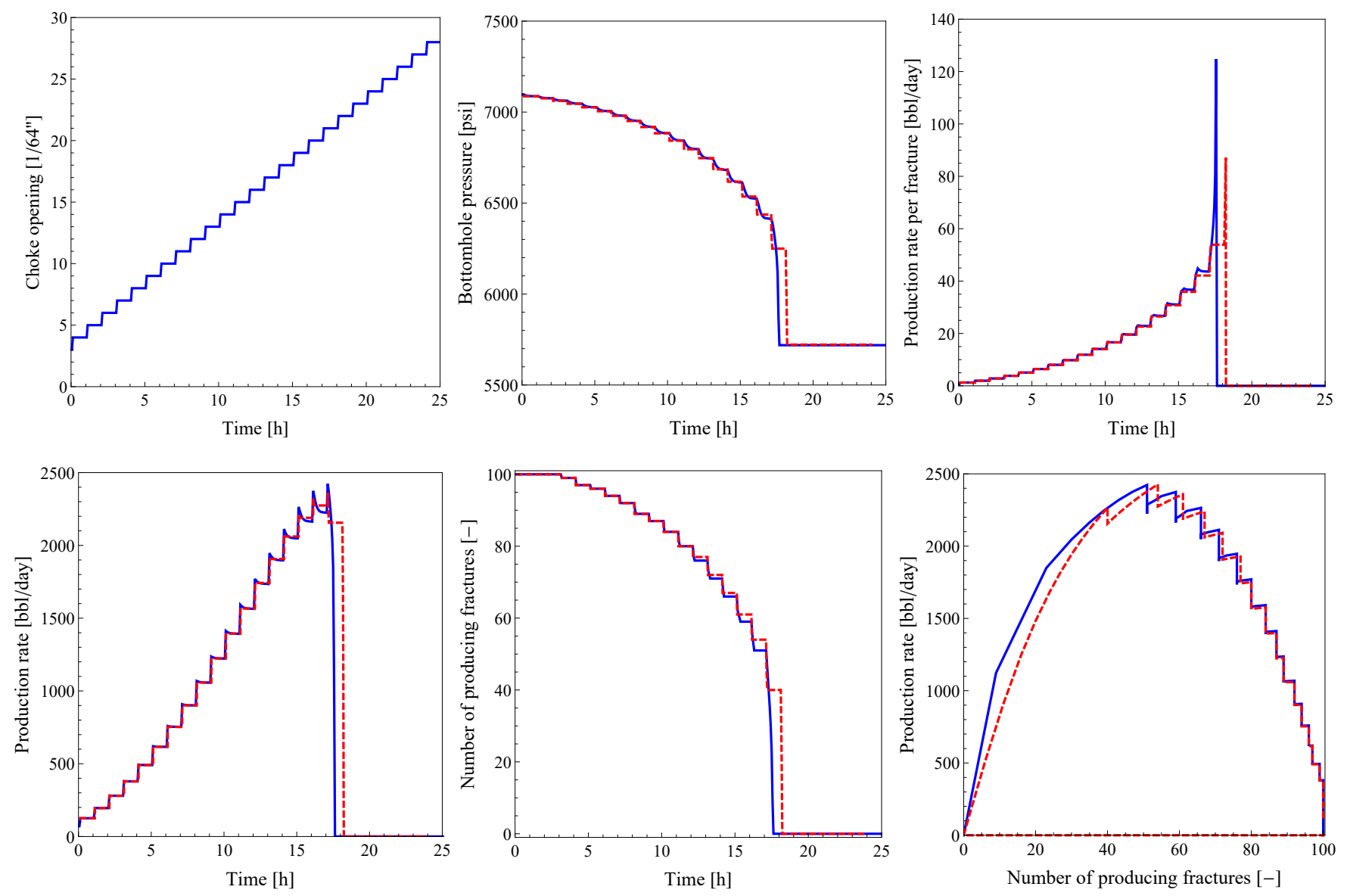

Figure 6: The results of numerical simulation of a cascading fracture failure in a multi-staged fractured well: gradual choke size change over the first 25 hours of flowback (top left); associated bottom-hole pressure drop (top middle); production rate from one fracture in the well (top right); total production rate from all fractures in the well (bottom left); number of producing fractures left in the well (bottom middle); production rate from the well (bottom right). Blue solid lines are the numerical simulation results. Red dashed lines are the analytical model predictions.

ture velocities would be equivalent to evolution of expected values of variables predicted by statistical approach.

Figure 6 illustrates the dynamics of choke size change,505 bottomhole pressure, production rate, and number of surviving producing fractures during the first 25 hours of flowback. Each step of choke opening is associated with a stepwise decrease of bottomhole pressure and stepwise increase of production rate. This corresponds to vertical segments510 on the charts representing pressures and flow rates as functions of time and number of fractures. More specifically, on the chart of total production rate as function of the number of production fractures, each timestep is denoted by a marker. After the production rate rises vertically515 due to a stepwise choke increase, the curve goes left and downward due to the failure of individual fractures. The reduced number of producing fractures redistributes the flow rates, which can cause further reduction in the number of producing fractures at the next timesteps and this520 cascade of fracture failures continues until the stable number of fractures is found or all fractures lose conductivity. In this case, the total production rate reaches its peak at about 50 producing fractures. Soon after that, there is the last stable combination of the choke size, rate, and number of fractures. The next increase of the choke size leads to the fast failure of the rest of the producing fractures in the final cascading event until all fractures in the well are completely closed and production is terminated. Results of the numerical simulation are in good agreement with the analytical model predictions.

Figure 7 demonstrates the total production rate versus number of producing fractures obtained with numerical model, analytical model, statistical description and Monte-Carlo simulations. Analytical model is capable of ultrafast runs, which makes it useful for the Monte-Carlo simulations. Monte-Carlo (MC) simulations were built by running the analytical model $4 \cdot 10^{6}$ times with uniform random distribution of critical fracture rates in the same range as in the statistical model. In the MC simulations, the choke was gradually opened in the same range from $3 / 64$ " to $28 / 64$ " with the finer step of $1 / 6400$ ". Then, for each combination of the choke setting and observed number of producing fractures, we accumulated number of tra- 
jectories crossing that point. For each given choke openof the observed number of producing fractures and calculate average number of producing fractures $\mu_{k}\left(d_{c h}\right)=$ $\sum_{k=1}^{N} n_{k}\left(d_{c h}\right) k$. Using the density distribution of the number of producing fractures, it is straightforward to calculate average production rate $\mu_{Q}\left(d_{c h}\right)=\sum_{k=1}^{N} n_{k}\left(d_{c h}\right) Q_{k}\left(d_{c h}\right)$ which corresponds to certain $\mu_{k}\left(d_{c h}\right)$. Here $Q_{k}$ is the flow rate delivered by $k$ producing fractures at given choke defined by the analytical model. Figure 7 essentially plots the average flow rate versus average number of producing fractures parameterized by the same value of choke opening.

Comparison of numerical, analytical, statistical and Monte-Carlo calculations represented in Figure 7 shows that there is good agreement between all of them. We would argue that all of them can be used interchangeably as needed and as allowed by the requirements of the problem that needs to be analyzed. This can be useful for engineering purposes, when, for example, it is necessary to analyze details of the mechanism of fracture conductivity damage inside fractures for some specific realization of fracture properties. The statistical or Monte-Carlo approach can be used first to identify the specific scenario or the trajectory in the total rate versus choke opening space. For example, one might focus on the lowest or highest 25th percentile in the rate distribution as function of choke. Then, the analytical model can be used to convert the selected rate versus choke trajectory into the specific realization of critical rates distribution that reproduce it. The latter can be simulated with the numerical model to analyze details of the fracture damage mechanisms and investigate options to re-engineer fracture properties.

As a final remark, we would add that the highly ideal-580 ized criterion of the immediate and complete fracture failure after the critical production rate is reached, has been adopted in the present work to clearly demonstrate effect of cascading failure introduced for the first time. In reality, one can expect less dramatic change of fracture conductiv-585 ity caused, for example, by the proppant flowback in the near-wellbore zone. Although the analytical study of this process is challenging, numerical simulations can provide insight in this case as well. The numerical study of the cascading failure for the more realistic configuration has590 been performed in [26], where the authors demonstrated that the cascading failure phenomenon can be observed in the simulations with gradual fracture conductivity degradation due to proppant mobilization.

\section{Effect of variable productivity indices of frac- tures}

The analytical derivations presented in the previous sections are based on the assumption of equal productivity ${ }^{600}$ index for all fractures. However, field observations (for example, [28]) indicate that productivity of fractures within

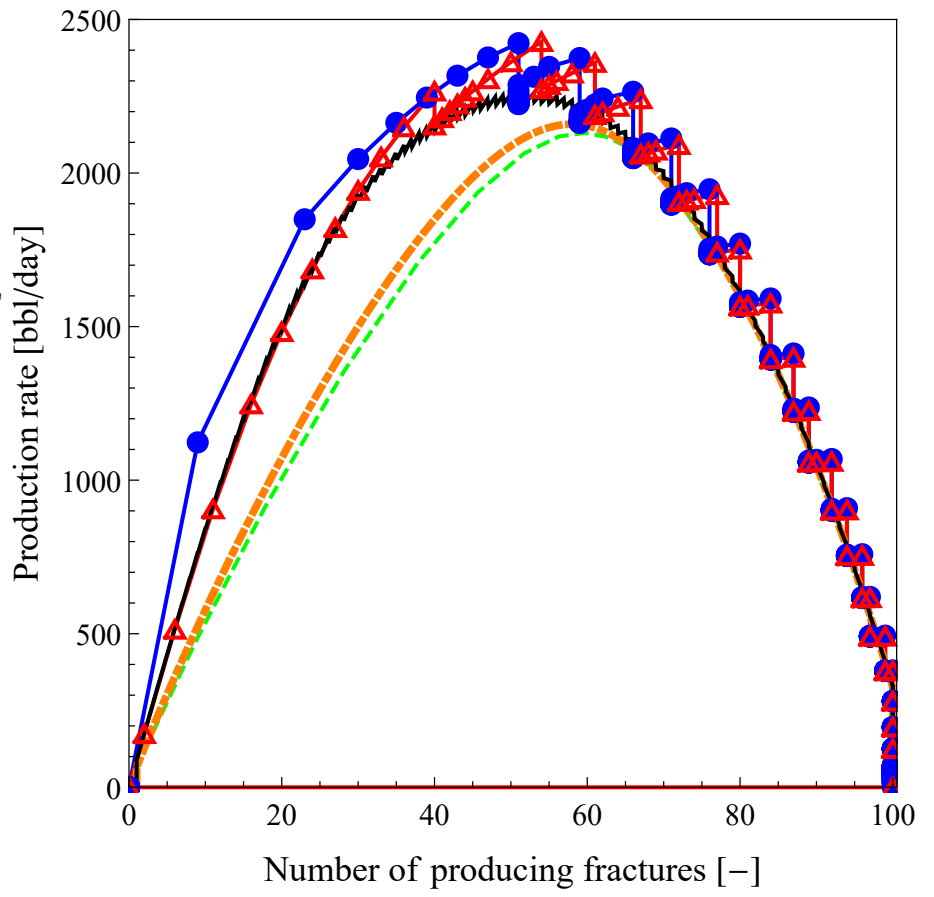

Figure 7: Comparison of simulation results obtained by different methods: rate versus number of fractures by numerical model with uniform distribution of critical fracture rates (blue disks), rate versus number of fractures by analytical model with uniform distribution (red triangles), average rate versus average number of fractures by statistical model (dashed green), average rate versus average number of fractures by Monte-Carlo model (dash-dotted thick orange), rate versus average number of fractures by Monte-Carlo model (solid black).

the well can be highly variable. The root cause of the cascading phenomenon is the flow rate redistribution between the remaining fractures following the failure of the particularly weak ones. Therefore, the existence of redistribution and cascading failure doesn't depend on whether the assumption of constant productivity index and is violated or not. The quantitative results of analysis can depend on details of productivity distributions among fractures. The details of productivity distribution are highly variable depending on geological context and rapidly changing operational practices. Collecting and analyzing such data deserves a separate study that beyond the scope of this paper. In this section, we evaluate whether the introduction of the simple non-uniform distribution of fracture productivity can bring significant changes to observations made above with constant productivity distribution.

A series of Monte-Carlo simulations has been performed to illustrate implications of productivity index variability. The assumption of uncorrelated productivity and critical rate has been adopted, such that the productivity indexes and critical flow rates of the fractures have been chosen from uniform distributions on the intervals $\left(J_{\min }, J_{\max }\right)$ and $\left(q_{\min }, q_{\max }\right)$. The problem of mutual correlation between productivity and stability of the fracture deserves separate study beyond the scope of this paper. The prob- 

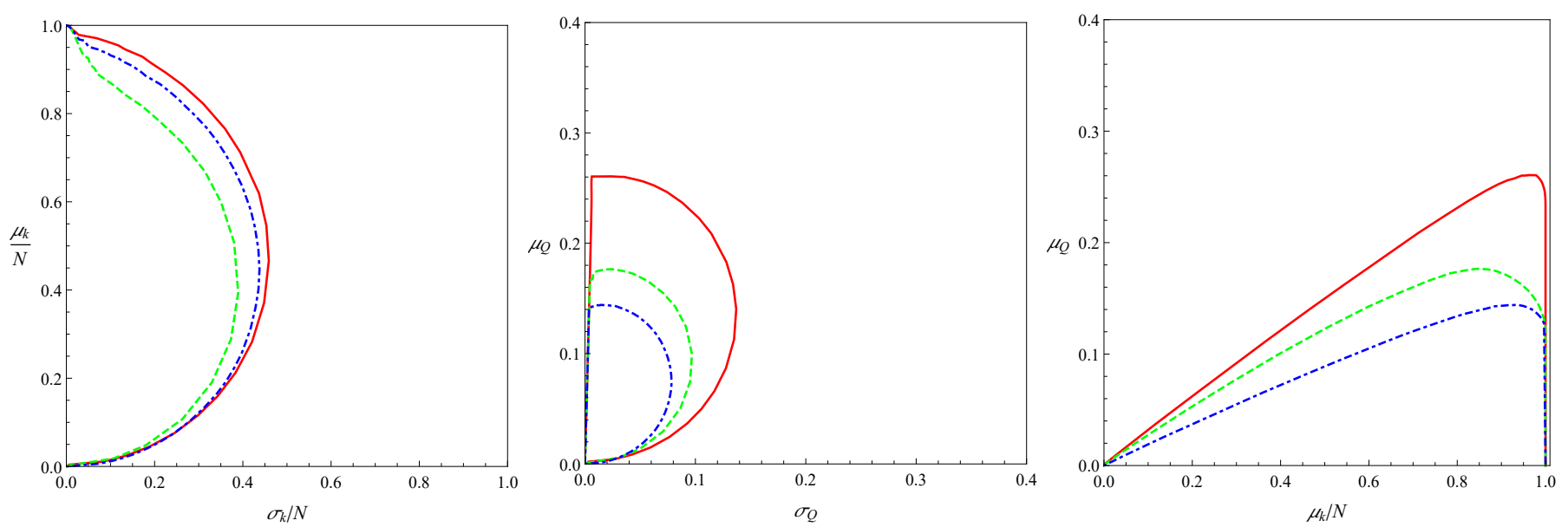

Figure 8: Parametric plots mean value vs. dispersion for the number of producing fractures (left) and the total production rate (center), the mean rate vs. mean number of producing fractures (right) with variable fracture productivity index. The parameters used are given in Table 1 , cases 1 (solid red), 2 (dashed green), and 3 (dash-dotted blue), $J_{\max } / J_{\min }=3$.
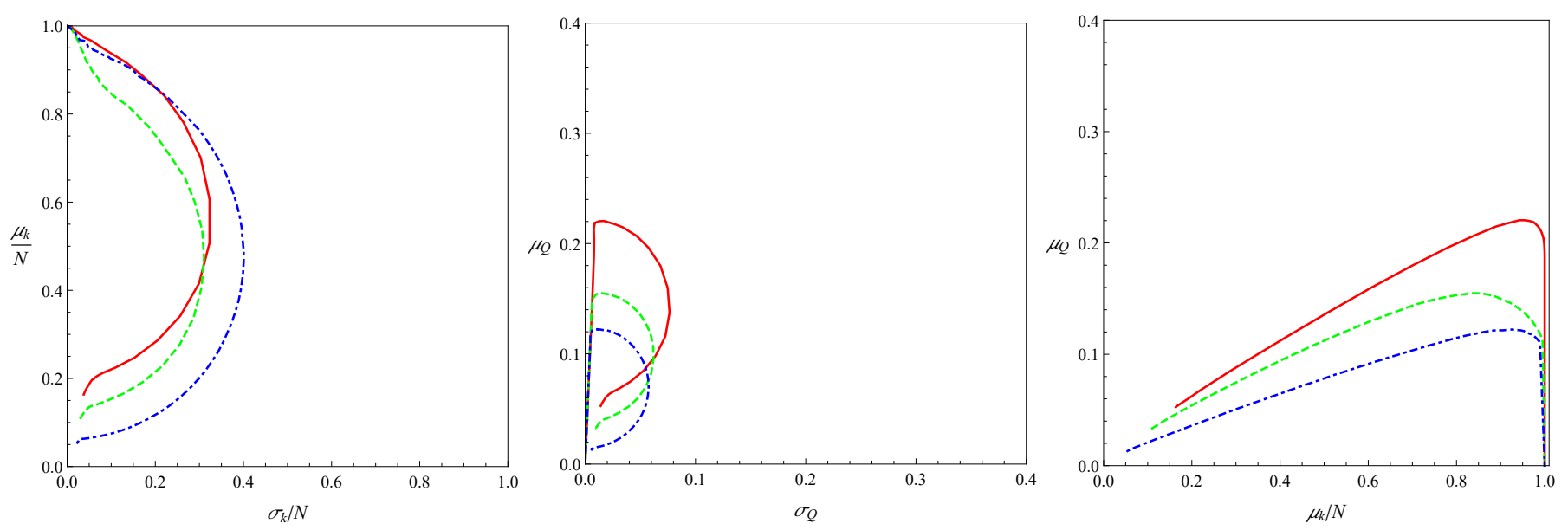

Figure 9: Parametric plots mean value vs. dispersion for the number of producing fractures (left) and the total production rate (center), the mean rate vs. mean number of producing fractures (right) with variable fracture productivity index. The parameters used are given in Table 1, cases 1 (solid red), 2 (dashed green), and 3 (dash-dotted blue), $J_{\max } / J_{\min }=10$.

lems with the same average productivity index as in the cases discussed above (see Table 1) has been studied to have a reference point for comparison. The ratio $J_{\max } / J_{\min }$ is considered as a measure of productivity index variability. Effectively, the fractures with productivity less than ${ }_{625}$ $J_{\text {min }}$ are excluded from consideration.

Results of the Monte-Carlo simulations for $J_{\max } / J_{\min }=$ 3 and $J_{\max } / J_{\min }=10$ are shown in Figure 8, 9. Scales for the plots are as in Figure 4 for the sake of visual comparison simplicity. For the lower ratio $J_{\max } / J_{\min }=3$ the 6 6о parametric plots $\left(\mu_{k}, \sigma_{k}\right)$ is similar to observed for equal productivity indexes (Figure 8, left). For the higher variability $J_{\max } / J_{\min }=10$, however, changes are significant. The expected number of producing fractures doesn't reach zero for maximum choke opening (Figure 9, left), because 635 the higher range of productivity leads to much broader range of critical pressures and, for some fraction of frac- tomhole pressure corresponding to fully open choke. Expected total production rate is not zero for fully open choke as well (Figure 9, center, right).

The magnitudes of the flow rate expected value and dispersion are remarkably lower than in the case of constant productivity index for both values of $J_{\max } / J_{\min }$ ratio (compare Figure 4, 8, 9, center). The reason of this effect is that, despite the assumption of zero correlation between critical rate and productivity, the critical pressure and productivity are negatively correlated accordint to the critical pressure definition (6). In other words, the most productive fractures are the least stable in terms of critical pressure. Therefore, these fractures fail first with increase of choke opening and contribute to steeper total rate reduction.

Finally, one can note that the main observation that was made based on the problems with constant productivity is valid as well in case of the variable productivity 
index of fractures. In both cases, the expected value of amount of fractures is already damaged (compare case 2 in Figure 4, right and Figure 8, 9, right). The variable productivity of fractures seems to enhance this effect. For example, in cases 1, 3, the decrease of mean total producing fractures is monotonic with constant productivity of fractures. With variable productivity of fractures, these cases demonstrate maximum flow rate for the fraction of non-damaged fractures slightly less than unity (compare cases 1 and 3 in Figure 4, right and Figure 8, 9 , right).

\section{Conclusion}

In this work, the emergent behaviour leading to the formation of the cascading failure mechanism in multistage hydraulically fractured wells during well startup and initial production is described and investigated. This phenomenon can be explained by existence of a feedback loop due to the hydraulic connection between individual producing fractures connected to the same wellbore. In our study, we presented several possible scenarios of evolution of cascading failure. The failure of weaker hydraulic fractures in such systems might increase drawdown on the stronger fractures, which survive. In turn, some of surviving fractures are weaker than others, so further increase of drawdown might lead to the cascading failure of an everincreasing amount of fractures. We also evaluated specific conditions triggering the cascading failure effect. To the best of our knowledge, the phenomenon of cascading failure of hydraulic fractures has not been studied in the previously published literature.

The hydraulic fracturing process is associated with a lack of robust measurement techniques and hence it is intrinsically uncertain. This provides grounds for treating the hydraulically fractured systems using a probabilistic approach and assuming that the failure criterion is reprerandom variable. Based on the proposed description, the analysis of the full system behavior is carried out. As an outcome, several choke opening strategies during the well startup could are formulated. Overall, these strategies deal with the trade-off between the short-term goal of maximizing production and long-term goal of preserving a larger number of hydraulic fractures. It is shown that moderate choke openings favor the well operating in the safe zone with productivity of all fractures preserved at the cost of relatively low production rates. Intermediate values of choke opening might lead to damage of some fractures; however, the production rates will be higher. Finally, the aggressive and large choke opening in extreme cases can lead to damage of all fractures. However, if some fractures survive with this strategy, a high production rate could be achieved. For the particular strategy aiming to maximize production rate, we propose a way to calculate choke management policy taking into account the statistical nature of fractures properties. Given the current number of producing fractures and choke opening, the policy defines the next choke adjustment maximizing the expected value of the final flow rate.

Finally, the evolution of cascading failure is demonstrated using numerical simulations that are based on fewer assumptions than analytical model and probabilistic description. It is shown that numerical results coincide qualitatively and quantitatively with the probabilistic description for the system with large number of hydraulic fractures.

\section{Acknowledgements}

The authors are grateful to Schlumberger for permission to publish this work. 


\section{Appendix A. Calculation of prior probabilities}

First, let us make the following change of variables in Equation (13):

$$
\omega_{k}=F\left(p_{c}^{(k)}\right), \quad \pi_{k}=F\left(p_{b h}^{k}\right) .
$$

Next, the product of theta-functions in Equation (13) may be transformed to

$$
\begin{aligned}
& \theta\left(p_{b h}^{k}-p_{c}^{(k)}\right) \theta\left(p_{c}^{(k+1)}-p_{b h}^{k}\right) \prod_{i=k+1}^{N}\left[\theta\left(p_{c}^{(i)}-p_{b h}^{i}\right)+\theta\left(p_{b h}^{i}-p_{c}^{(i+1)}\right)\right]= \\
& =\theta\left(\pi_{k}-\omega_{k}\right) \theta\left(\omega_{k+1}-\pi_{k}\right) \prod_{i=k+1}^{N}\left[\theta\left(\omega_{i}-\pi_{i}\right)+\theta\left(\pi_{i}-\omega_{i+1}\right)\right]= \\
& =\theta\left(\pi_{k}-\omega_{k}\right) \theta\left(\omega_{k+1}-\pi_{k}\right) \prod_{i=k+1}^{N-2}\left[\theta\left(\omega_{i}-\pi_{i}\right)+\theta\left(\pi_{i}-\omega_{i+1}\right)\right] \times \\
& =\theta\left(\pi_{k}-\omega_{k}\right) \theta\left(\omega_{k+1}-\pi_{k}\right) \prod_{i=k+1}^{N-2}\left[\theta\left(\omega_{N-1}-\pi_{N-1}\right)+\theta\left(\pi_{N-1}-\omega_{N}\right)\right] \theta\left(\omega_{N}-\pi_{N}\right)= \\
& \left.\quad \times \theta\left(\pi_{i}-\omega_{i+1}\right)\right] \times \\
& =\theta\left(\pi_{k}-\omega_{k}\right) \prod_{i=k+1}^{N} \theta\left(\omega_{i}-\pi_{i}\right)
\end{aligned}
$$

The latter transformation takes into account that $\pi_{k+1}>\pi_{k}$ and consequently

$$
\theta\left(\pi_{k-1}-\omega_{k}\right) \theta\left(\omega_{k}-\pi_{k}\right) \equiv 0 .
$$

Then, using Equation (12) one can obtain

$$
\begin{gathered}
P_{k}=\underbrace{\int_{0}^{1} \cdots \int_{0}^{1}}_{N-k+1} \prod_{i=k}^{N} d \omega_{i} \theta\left(\omega_{i+1}-\omega_{i}\right) \theta\left(\pi_{k}-\omega_{k}\right) \prod_{i=k+1}^{N} \theta\left(\omega_{i}-\pi_{i}\right) \frac{N !}{(k-1) !} \omega_{k}^{k-1}= \\
=\int_{0}^{1} d \omega_{N} \theta\left(\omega_{N}-\pi_{N}\right) \underbrace{\int_{0}^{1} \cdots \int_{0}^{1}}_{N-k} \prod_{i=k}^{N-1} d \omega_{i} \theta\left(\omega_{i+1}-\omega_{i}\right) \theta\left(\pi_{k}-\omega_{k}\right) \prod_{i=k+1}^{N-1} \theta\left(\omega_{i}-\pi_{i}\right) \frac{N !}{(k-1) !} \omega_{k}^{k-1} .
\end{gathered}
$$

Rearranging integration order one can get

$$
\begin{aligned}
P_{k}=\frac{N !}{(k-1) !} & \int_{0}^{1} d \omega_{N} \int_{0}^{1} d \omega_{N-1} \theta\left(\omega_{N}-\pi_{N}\right) \theta\left(\omega_{N}-\omega_{N-1}\right) \times \cdots \times \\
& \times \int_{0}^{1} d \omega_{k+1} \theta\left(\omega_{k+2}-\pi_{k+2}\right) \theta\left(\omega_{k+2}-\omega_{k+1}\right) \times \\
& \times \int_{0}^{1} d \omega_{k} \omega_{k}^{k-1} \theta\left(\omega_{k+1}-\pi_{k+1}\right) \theta\left(\omega_{k+1}-\omega_{k}\right) \theta\left(\pi_{k}-\omega_{k}\right)= \\
=\frac{N !}{k !} & \pi_{k}^{k} \int_{0}^{1} d \omega_{N} \theta\left(\omega_{N}-\pi_{N}\right) \int_{0}^{1} d \omega_{N-1} \theta\left(\omega_{N-1}-\pi_{N-1}\right) \theta\left(\omega_{N}-\omega_{N-1}\right) \times \cdots \times \\
& \times \int_{0}^{1} d \omega_{k+2} \theta\left(\omega_{k+3}-\omega_{k+2}\right) \theta\left(\omega_{k+2}-\pi_{k+2}\right) \times \\
& \times \int_{0}^{1} d \omega_{k+1} \theta\left(\omega_{k+2}-\omega_{k+1}\right) \theta\left(\omega_{k+1}-\pi_{k+1}\right)= \\
\frac{N !}{k !} & \pi_{k}^{k} \int_{0}^{1} d \omega_{N} \theta\left(\omega_{N}-\pi_{N}\right) \int_{0}^{1} d \omega_{N-1} \theta\left(\omega_{N-1}-\pi_{N-1}\right) \theta\left(\omega_{N}-\omega_{N-1}\right) \times \cdots \times \\
& \times \int_{0}^{1} d \omega_{k+2} \theta\left(\omega_{k+3}-\omega_{k+2}\right) \theta\left(\omega_{k+2}-\pi_{k+2}\right) \theta\left(\omega_{k+2}-\pi_{k+1}\right)\left(\omega_{k+2}-\pi_{k+1}\right) .
\end{aligned}
$$


The latter transformation takes again into account that $\pi_{k+1}>\pi_{k}$ and

$$
\theta\left(\omega_{k+1}-\pi_{k+1}\right) \theta\left(\omega_{k+1}-\pi_{k}\right) \equiv \theta\left(\omega_{k+1}-\pi_{k+1}\right) .
$$

Let us define a sequence of functions

$$
g_{k, l+1}\left(\omega_{l+1}\right)=\int_{0}^{1} d \omega_{l} \theta\left(\omega_{l+1}-\omega_{l}\right) \theta\left(\omega_{l}-\pi_{l}\right) g_{k, l}\left(\omega_{l}\right) .
$$

Here index $l=\overline{k+2, N+1}$ and the starting member of the sequence is

$$
g_{k, k+2}\left(\omega_{k+2}\right)=\theta\left(\omega_{k+2}-\pi_{k+1}\right)\left(\omega_{k+2}-\pi_{k+1}\right) .
$$

Using the previous definition, one can rewrite the equation for probability as

$$
P_{k}=\frac{N !}{k !} \pi_{k}^{k} \int_{0}^{1} d \omega_{N} \theta\left(\omega_{N}-\pi_{N}\right) g_{k, N}\left(\omega_{N}\right)=\frac{N !}{k !} \pi_{k}^{k} \theta\left(1-\pi_{N}\right) g_{k, N+1}(1) .
$$

One can check by the direct substitution into Equation (A.4) that the functions $g_{k, l}\left(\omega_{l}\right)$ are polynomials multiplied by appropriate theta function:

$$
g_{k, l}\left(\omega_{l}\right)=\theta\left(\omega_{l}-\pi_{l-1}\right) \sum_{i=0}^{l-k-1} a_{k, l, i} \omega_{l}^{i}, \quad l=\overline{k+2, N+1} .
$$

Coefficients of polynomials for successive indexes $l$ are related in the following way:

$$
a_{k, l+1, i+1}=\frac{a_{k, l, i}}{i+1}, \quad i=\overline{0, l-k-1}, \quad a_{k, l+1,0}=-\sum_{i=0}^{l-k-1} \frac{a_{k, l, i}}{i+1} \pi_{l}^{i+1} .
$$

Equation (A.8) and (A.5) allow recursive calculation of polynomial coefficients for $g_{k, N+1}$ and, thus, probability $P_{k}$ according to Equation (A.6).

\section{Appendix B. Calculation of conditional probabilities}

First, let us use again the change of variables (A.1) and the result (A.2) to transform the product of theta-functions in Equation (16)

$$
\begin{gathered}
\prod_{m=m_{0}}^{M} \theta\left(p_{b h}^{k_{m}}\left(C_{m}\right)-p_{c}^{\left(k_{m}\right)}\right) \theta\left(p_{c}^{\left(k_{m}+1\right)}-p_{b h}^{k_{m}}\left(C_{m}\right)\right) \prod_{i=k_{m}+1}^{N}\left[\theta\left(p_{c}^{(i)}-p_{b h}^{i}\left(C_{m}\right)\right)+\theta\left(p_{b h}^{i}\left(C_{m}\right)-p_{c}^{(i+1)}\right)\right]= \\
=\prod_{m=m_{0}}^{M} \theta\left(\pi_{k_{m}}\left(C_{m}\right)-\omega_{k_{m}}\right) \prod_{i=k_{m}+1}^{N} \theta\left(\omega_{i}-\pi_{i}\left(C_{m}\right)\right)
\end{gathered}
$$

Next, using the conditions $\pi_{i}\left(C_{m+1}\right)<\pi_{i}\left(C_{m}\right), \forall i, m$ and $k_{m} \leq k_{m+1}, \forall m$, one can transform the latter equation:

$$
\begin{aligned}
& \prod_{m=m_{0}}^{M} \theta\left(\pi_{k_{m}}\left(C_{m}\right)-\omega_{k_{m}}\right) \prod_{i=k_{m}+1}^{N} \theta\left(\omega_{i}-\pi_{i}\left(C_{m}\right)\right)= \\
= & \prod_{m=m_{0}}^{M} \theta\left(\pi_{k_{m}}\left(C_{m}\right)-\omega_{k_{m}}\right)\left[\prod_{i=k_{m}+1}^{k_{m+1}-1} \theta\left(\omega_{i}-\pi_{i}\left(C_{m}\right)\right)\right] \theta\left(\omega_{k_{m+1}}-\pi_{k_{m+1}}\left(C_{m}\right)\right) .
\end{aligned}
$$

Then, using Equation (12) one can get

$$
\begin{aligned}
P\left(\bigcap_{m=m_{0}}^{M} \mathcal{A}\left(k_{m}, C_{m}\right)\right)=\underbrace{\int \cdots \int}_{N-k_{m_{0}}+1} \prod_{i=k_{m_{0}}}^{N} d \omega_{i} \theta\left(\omega_{i+1}-\omega_{i}\right) \frac{N !}{\left(k_{m_{0}}-1\right) !} \omega_{k_{m_{0}}}^{k_{m_{0}}-1} \times \\
\quad \times \prod_{m=m_{0}}^{M} \theta\left(\pi_{k_{m}}\left(C_{m}\right)-\omega_{k_{m}}\right)\left[\prod_{i=k_{m}+1}^{k_{m+1}-1} \theta\left(\omega_{i}-\pi_{i}\left(C_{m}\right)\right)\right] \theta\left(\omega_{k_{m+1}}-\pi_{k_{m+1}}\left(C_{m}\right)\right) .
\end{aligned}
$$


Change of integration order gives

$$
\begin{aligned}
& P\left(\bigcap_{m=m_{0}}^{M} \mathcal{A}\left(k_{m}, C_{m}\right)\right)=\underbrace{\int \ldots \int}_{N-k_{m_{0}+1}+1} \prod_{i=k_{m_{0}+1}}^{N} d \omega_{i} \theta\left(\omega_{i+1}-\omega_{i}\right) \times \theta\left(\omega_{k_{m_{0}+1}}-\pi_{k_{m_{0}+1}}\left(C_{m_{0}}\right)\right) \times \\
& \quad \times \prod_{m=m_{0}+1}^{M} \theta\left(\pi_{k_{m}}\left(C_{m}\right)-\omega_{k_{m}}\right)\left[\prod_{i=k_{m}+1}^{k_{m+1}-1} \theta\left(\omega_{i}-\pi_{i}\left(C_{m}\right)\right)\right] \theta\left(\omega_{k_{m+1}}-\pi_{k_{m+1}}\left(C_{m}\right)\right) \times \\
& \times \underbrace{\int \ldots \int}_{k_{m_{0}+1}-k_{m_{0}}} \prod_{i=k_{m_{0}}}^{k_{m_{0}+1}-1} d \omega_{i} \theta\left(\omega_{i+1}-\omega_{i}\right) \theta\left(\pi_{k_{m_{0}}}\left(C_{m_{0}}\right)-\omega_{k_{m_{0}}}\right)] \prod_{i=k_{m_{0}}+1}^{k_{m_{0}+1}-1} \theta\left(\omega_{i}-\pi_{i}\left(C_{m_{0}}\right)\right)] \frac{N !}{\left(k_{m_{0}}-1\right) !} \omega_{k_{m_{0}}}^{k_{m_{0}}-1} .
\end{aligned}
$$

Comparing the last line of the previous equation with the relation for prior probability (A.3) and utilizing the definition given by Equation (A.4) one can obtain

$$
\begin{aligned}
& P\left(\bigcap_{m=m_{0}}^{M} \mathcal{A}\left(k_{m}, C_{m}\right)\right)=\underbrace{\int \ldots \int}_{N-k_{m_{0}+1}+1} \prod_{i=k_{m_{0}+1}}^{N} d \omega_{i} \theta\left(\omega_{i+1}-\omega_{i}\right) \frac{N !}{k_{m_{0}} !} \pi_{k_{m_{0}}}^{k_{m_{0}}}\left(C_{m_{0}}\right) g_{k_{m_{0}}, k_{m_{0}+1}}\left(\omega_{k_{m_{0}+1}} ; C_{m_{0}}\right) \times \\
& \quad \times \prod_{m=m_{0}+1}^{M} \theta\left(\pi_{k_{m}}\left(C_{m}\right)-\omega_{k_{m}}\right)\left[\prod_{i=k_{m}+1}^{k_{m+1}-1} \theta\left(\omega_{i}-\pi_{i}\left(C_{m}\right)\right)\right] \theta\left(\omega_{k_{m+1}}-\pi_{k_{m+1}}\left(C_{m}\right)\right) .
\end{aligned}
$$

Here the argument $C_{m_{0}}$ in the term $g_{k_{m_{0}}, k_{m_{0}+1}}\left(\omega_{k_{m_{0}+1}} ; C_{m_{0}}\right)$ shows that the values $\pi_{l}\left(C_{m_{0}}\right)$ have been used to construct the polynomial $g_{k_{m_{0}}, k_{m_{0}+1}}$ by formulas (A.7), (A.8).

Because Equation (B.1) and (B.2) differ only by the change of index $m_{0} \rightarrow m_{0}+1$ and replacement of the factor

$$
\frac{N !}{\left(k_{m_{0}}-1\right) !} \omega_{k_{m_{0}}}^{k_{m_{0}}-1} \rightarrow \frac{N !}{k_{m_{0}} !} \pi_{k_{m_{0}}}^{k_{m_{0}}}\left(C_{m_{0}}\right) g_{k_{m_{0}}, k_{m_{0}+1}}\left(\omega_{k_{m_{0}+1}} ; C_{m_{0}}\right)
$$

it is possible to repeat the calculation exactly in the same way and finally get

$$
P\left(\bigcap_{m=m_{0}}^{M} \mathcal{A}\left(k_{m}, C_{m}\right)\right)=\frac{N !}{k_{m_{0}} !} \pi_{k_{m_{0}}}^{k_{m_{0}}}\left(C_{m_{0}}\right) \prod_{m=m_{0}}^{M} g_{k_{m}, k_{m+1}+1}\left(\pi_{k_{m+1}+1}\left(C_{m+1}\right) ; C_{m}\right) .
$$

Here $k_{M+1}=N, \pi_{N+1}=1$.

Substitution of this result to Equation (15) leads to

$$
P_{\left(k_{0}, C_{0}\right) \mid\left(k_{1}, C_{1}\right), \ldots,\left(k_{M}, C_{M}\right)}=\frac{k_{1} !}{k_{0} !} \frac{\pi_{k_{0}}^{k_{0}}\left(C_{0}\right)}{\pi_{k_{1}}^{k_{1}}\left(C_{1}\right)} g_{k_{0}, k_{1}+1}\left(\pi_{k_{1}+1}\left(C_{1}\right) ; C_{0}\right)=P_{\left(k_{0}, C_{0}\right) \mid\left(k_{1}, C_{1}\right)}
$$

Surprisingly, the conditional probability depends only on the last observed state of the well. Probably, there exist a more elegant (than direct calculation presented here) way to prove this fact.

\section{References}

[1] A. V. Brovchuk, I. Diyashev, A. V. Lipyanin, D. Grant, D. Oussoltsev, K. K. Butula, Fracturing Treatments of Openhole Horizontal Wells in Western Siberia, in: SPE Russian Oil and Gas Technical Conference and Exhibition, 3-6 October, 2006,735 Moscow, Russia, Society of Petroleum Engineers, 2006. doi: 10.2118/102417-RU.

[2] M. Stanojcic, O. A. Jaripatke, A. Sharma, Pinpoint Fracturing Technologies: A Review of Successful Evolution of Multistage Fracturing in the Last Decade, in: SPE/ICoTA Coiled Tubing740 and Well Intervention Conference and Exhibition, 23-24 March, 2010, The Woodlands, Texas, USA, Society of Petroleum Engineers, 2010. doi:10.2118/130580-MS.

[3] A. A. Daneshy, Hydraulic Fracturing of Horizontal Wells: Is- sues and Insights, in: SPE Hydraulic Fracturing Technology Conference, 24-26 January, 2011, The Woodlands, Texas, USA, Society of Petroleum Engineers, 2011. doi:10.2118/140134-MS.

[4] M. C. Vincent, Optimizing Transverse Fractures in Liquid-Rich Formations, in: SPE Annual Technical Conference and Exhibition, 30 October-2 November, 2011, Denver, Colorado, USA, Society of Petroleum Engineers, 2011. doi:10.2118/146376-ms.

[5] R. Baldick, B. Chowdhury, I. Dobson, Z. Dong, B. Gou, D. Hawkins, H. Huang, M. Joung, D. Kirschen, F. Li, J. Li, Z. Li, C. C. Liu, L. Mili, S. Miller, R. Podmore, K. Schneider, K. Sun, D. Wang, Z. Wu, P. Zhang, W. Zhang, X. Zhang, Initial review of methods for cascading failure analysis in electric power transmission systems, in: IEEE Power and Energy Society 2008 General Meeting: Conversion and Delivery of Electrical Energy in the 21st Century, PES, 20-24 July, 2008, Pittsburgh, PA, 
SA, IEEE, 2008, doi:10.1109/PES. 2008.4596430.

[6] J. Song, E. Cotilla-Sanchez, G. Ghanavati, P. Hines, Dynamic modeling of cascading failure in power systems, IEEE Trans-790 actions on Power Systems 31 (2016) 2085-2095. doi:10.1109/ TPWRS. 2015. 2439237.

[7] H. Guo, C. Zheng, H. H.-C. Iu, T. Fernando, A critical review of cascading failure analysis and modeling of power system, Renewable and Sustainable Energy Reviews 80 (2017) 9-795 22. doi:10.1016/j.rser.2017.05.206.

[8] C. Ellinas, N. Masuda, Modelling project failure and its mitigation in a time-stamped network of interrelated tasks (2018). arXiv: 1810.07561

[9] Q. Shuang, M. Zhang, Y. Yuan, Node vulnerability of wa-800 ter distribution networks under cascading failures, Reliability Engineering \& System Safety 124 (2014) 132-141. doi: 10.1016/j.ress. 2013.12.002.

[10] A. Yazdani, P. Jeffrey, Complex network analysis of water distribution systems, Chaos: An Interdisciplinary Journal of Non-805 linear Science 21 (1) (2011) 016111. doi:10.1063/1.3540339.

[11] Y. Wang, H. Huang, B. Su, Vulnerability analysis of urban natural gas network under cascading failures, in: 2015 International Conference on Industrial Technology and Management Science, Atlantis Press, 2015. doi:10.2991/itms-15.2015.146.

12] A A Osiptsov, E. Zilonova, S. Boronin, J. Desroches, N. Lebedeva, D. Willberg, Insights on Overflushing Strategies from a Novel Modeling Approach to Displacement of Yield-Stress Fluids in a Fracture, in: SPE Annual Technical Conference and Exhibition, 26-28 September, 2016, Dubai, UAE, Society of 815 Petroleum Engineers, 2016. doi:10.2118/181454-MS.

[13] R. Sachdeva, Z. Schmidt, J. Brill, R. Blais, Two-Phase Flow Through Chokes, in: SPE Annual Technical Conference and Exhibition, 5-8 October, 1986, New Orleans, Louisiana, Society of Petroleum Engineers, 1986. doi:10.2523/15657-MS.

[14] M. Economides, K. Nolte, Reservoir Stimulation, Third Edition, John Wiley \& Sons, 2000.

[15] P. Robin, Note on effective pressure, Journal of Geophysical Research 78 (1973) 2434-2437. doi:10.1029/JB078i014p02434.

[16] Y. Bernabe, The effective pressure law for permeability in825 chelmsford granite and barre granite, International Journal of Rock Mechanics and Mining Sciences \& Geomechanics Abstracts 23 (1986) 267-275. doi:10.1016/0148-9062(86) 90972-1.

[17] W. Xiao, Y. Bernabe, B. Evans, U. Mok, J. Zhao, X. Ren,8зо
M. Chen, Klinkenberg effect and effective pressure for gas permeability of tight sandstones, Journal of Geophysical Research: Solid Earth 124 (2019) 1412-1429. doi:10.1029/2018JB016686.

[18] M. Ahsanullah, V. B. Nevzorov, M. Shakil, An Introduction to Order Statistics, Atlantis Studies in Probability and Statistics 3, Atlantis Press, 2013. doi:10.2991/978-94-91216-83-1_2.

[19] R. A. Howard, Dynamic programming and Markov processes., John Wiley, 1960

[20] M. L. Puterman, Markov decision processes: discrete stochastic dynamic programming, John Wiley \& Sons, 1994.

[21] R. Bellman, A Markovian decision process, Journal of Mathematics and Mechanics (1957) 679-684.

URL http://www.jstor.org/stable/24900506

[22] P. Spesivtsev, A. Kharlashkin, K. Sinkov, Study of the transient terrain-induced and severe slugging problems by use of the drift-flux model, SPE Journal 22 (2017) 1570-1584. doi: 10.2118/186105-PA.

[23] D. Chuprakov, L. Belyakova, A. Iuldasheva, A. Alekseev, D. Syresin, M. Chertov, P. Spesivtsev, F. I. Salazar Suarez, I. Velikanov, L. Semin, D. Bannikov, Proppant Flowback: Can We Mitigate the Risk?, in: SPE Hydraulic Fracturing Technology Conference and Exhibition, 4-6 February, 2020, The Woodlands, TX, USA, Society of Petroleum Engineers, 2020. doi:10.2118/199748-MS.

[24] S. Whitaker, Flow in porous media I: A theoretical derivation of Darcy's law, Transport in Porous Media 1 (1986) 3-25. doi:10.1007/BF01036523.

[25] E. Isaacson, H. Keller, Analysis of numerical methods, Dover, 1994.

[26] M. Chertov, K. Sinkov, On numerical simulation of cascading failure of hydraulic fractures due to aggressive flowback, ARMA20-1637, in: 54th US Rock Mechanics/Geomechanics Symposium, 28 June - 1 July, 2020, Golden, Colorado, USA, 2020

[27] V. Aksenov, M. Chertov, K. Sinkov, Application of accelerated fixed-point algorithms to hydrodynamic well-fracture coupling (2020). arXiv:2005.01620.

[28] R. W. Slocombe, A. M. Acock, K. Fisher, A. Viswanathan, C. Chadwick, R. L. Reischman, E. Wigger, Eagle ford completion optimization using horizontal log data, in: SPE Annual Technical Conference and Exhibition, 30 September-2 October, 2013, New Orleans, Louisiana, USA, Society of Petroleum Engineers, 2013. doi:10.2118/166242-MS. 